\title{
Ex vivo Approach to Treat Failing Organs: Expanding the Limits
}

\author{
Anatol Ciubotaru ${ }^{a, b} \quad$ Axel Haverich ${ }^{a}$ \\ ${ }^{a}$ Department of Cardiothoracic, Transplantation and Vascular Surgery,

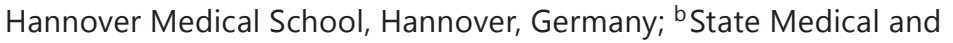 \\ Pharmaceutical University 'Nicolae Testemitanu', Chisinau, Republic of Moldova
}

\section{Key Words}

Ex vivo organ perfusion - Ex vivo organ preservation - Ex vivo organ treatment .

Autotransplantation

\begin{abstract}
Background: Advanced organ failure is often classified as an end-stage disease where the treatment options are limited only to transplantation. As an alternative, different attempts have been undertaken to improve the outcome of the treatment of failing organs by using targeted ex vivo approaches. This may solve the issue of organ shortage by treating the donor organs before transplantation and the number of patients requiring transplantation may also be reduced by applying extensive ex vivo treatment followed by autotransplantation. Methods: We performed a literature review of PubMed and included articles published between 1962 and 2013. The following keywords were used (and; or): ex vivo, therapy, surgery, organ perfusion and autotransplantation. This review includes specific methods and attempts related to ex vivo organ perfusion and preservation, temporary life support systems, surgical and other therapeutic approaches, and diagnostic methods applied ex vivo to an isolated organ. Results: For the practical clinical use of ex vivo therapies, we could identify three major directions: (1) ex vivo pretransplant organ reconditioning, (2) ex vivo surgery and (3) ex vivo medical treatment. Different attempts have been made worldwide in the above-mentioned areas focusing on ex vivo organ preservation and treatment. We summarize in the present review the developments in the field of ex vivo organ recovery and evaluate the possibilities of combining and applying different technologies such as organ perfusion and storage, ex vivo exact topographical diagnosis, ex vivo locoregional medical treatment and ex vivo surgical correction. Conclusion: Ex vivo therapies open new horizons in the treatment of end-stage organ pathologies.




\section{Introduction}

Organ transplantation remains the final solution for patients with end-stage organ failure. However, the limited number of physiologically functional donor organs causes a continuous increase in the number of patients on the waiting lists for transplantation. Therefore, a large number of patients remains excluded from the option of transplantation [1,2].

In the last 5 years, the system for extracorporeal membrane oxygenation has undergone a significant development, bringing safety and efficacy to the treatment of patients with advanced cardiorespiratory disorders [3]. Extracorporeal membrane oxygenation can even be used in awake, nonintubated patients, bridging the time till they receive a donor organ, and it can also give the patients' own diseased organs time for partial or complete recovery $[4,5]$. Furthermore, different organ perfusion systems have been developed and implemented in clinical practice in order to maintain the viability of organs ex vivo [6].

The priorities of ex vivo therapies are the following:

- Ex situ topical surgical assessment of the diseased organ, which may allow precise excision and/or reconstruction of the tissue.

- Ex situ topical imaging for diagnosis based on exact assessment of the diseased tissue and precise definition of the borderlines of the healthy area.

- Local application of the medication or local exposure to radiotherapy allowing the application of high dosages and thus avoiding systemic application of the treatment.

- Ex vivo resuscitation of the failing organ by using improved perfusion in an optimized environment $(\mathrm{pH}$, temperature, etc.) and preventing toxic metabolites from disseminating into the systemic circulation.

- Ex vivo pretransplant assessment and preservation of the organ.

For practical clinical use, ex vivo therapies are oriented in three major directions (fig. 1):

(1) ex vivo pretransplant organ reconditioning, (2) ex vivo surgery and (3) ex vivo medical treatment.

In this review we aim to summarize the developments in the field of ex vivo organ recovery and to discuss the possibilities of combining and applying different technologies to address the effectiveness of isolated treatments of failed organs.

\section{Ex vivo Pretransplant Organ Reconditioning}

The number of patients in need of a donor organ constantly exceeds the number of organ donations. Yet, only 15-25\% of the lungs from donors donating at least one other organ are transplanted, representing the lowest graft acceptance rate [7]. Very often the criteria for a 'marginal' organ differ and/or criteria are applied differently by different transplant teams. Suboptimal assessment of organ function often leads to rejection of the donor tissue.

Progress in organ transplant technologies has led to the development of organ perfusion systems including portable devices that allow extended organ preservation and ex vivo perfusion. The Organ Care System (OCS) serves as an example. Developed by TransMedics (Andover, Mass., USA) for heart and lungs, it is a portable blood pulsatile perfusion system, which mimics physiological conditions and is designed to assess and improve marginal organs and to potentially improve the condition of routine donor organs [8].

Monbaliu and Brassil [9] have defined several advantages for organ preservation using machine perfusion compared with simple cold storage: (1) provision of continuous circulation and better preservation of the microcirculation, (2) continuous nutrient and oxygen delivery to fulfill the organ's metabolic demands, (3) removal of metabolic waste products and toxins, (4) opportunity to assess organ viability, (5) improved clinical outcomes by 


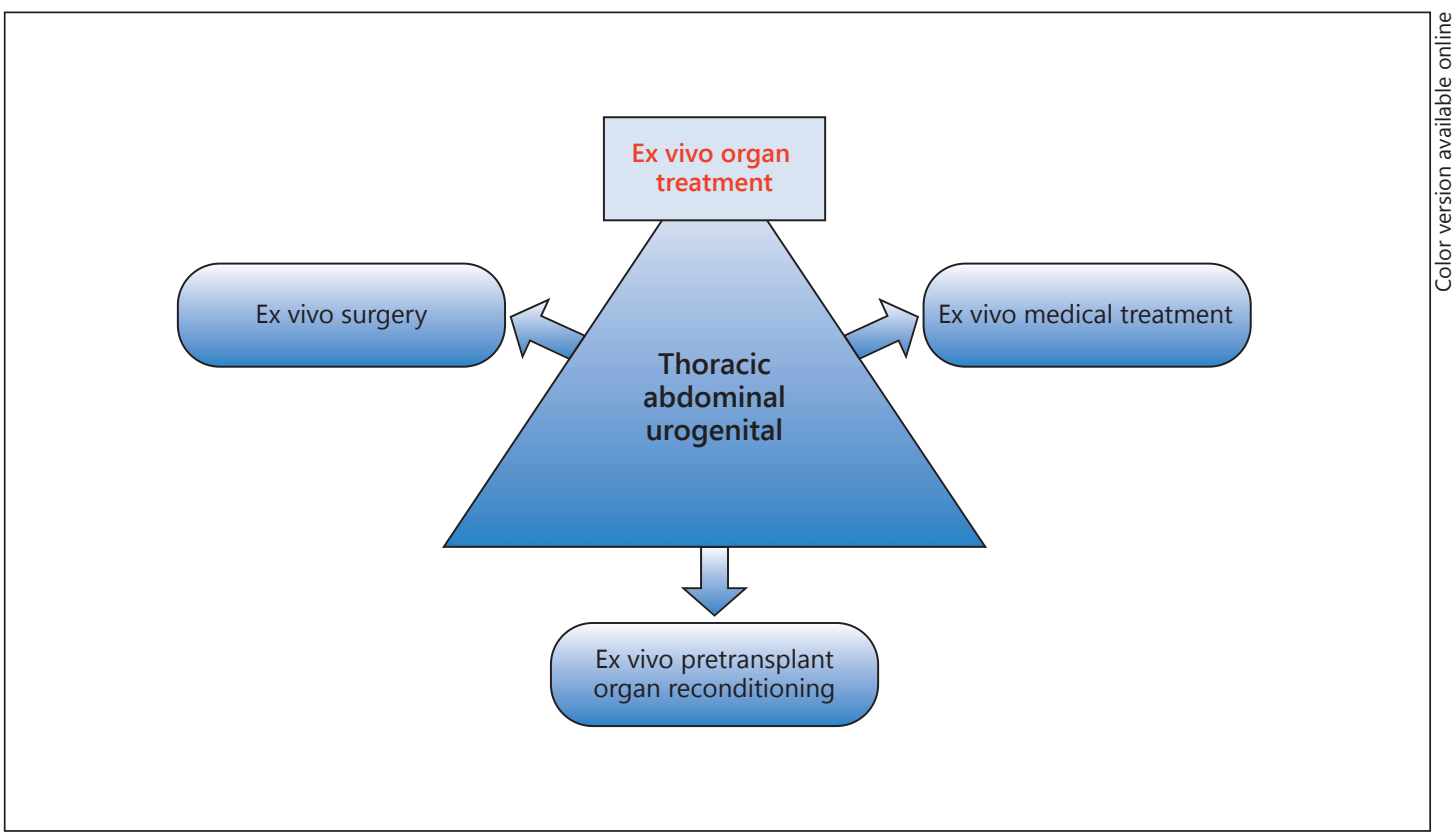

Fig. 1. Schematic illustration of ex vivo approaches in organ treatment.

improved immediate graft function rates, (6) prolonged preservation time without increased preservation damage, (7) administration of cytoprotective and immunomodulating substances, and (8) economic benefit due to a lower graft dysfunction incidence, shorter hospital stays and better graft survival rates.

Assessment of the organ condition can also be performed by using imaging tools, including $\mathrm{X}$-ray, CT or angiography, that can be used ex situ on an isolated organ.

\section{Lungs}

A group of surgeons from the Lund University in Sweden first successfully used human ex vivo perfusion for lung evaluation and modification [10]. They reported that 6 out of 9 initially rejected donor lungs were reconditioned to acceptable function, and in 6 recipients, double-lung transplantation was performed with a 3-month survival of $100 \%$.

Aigner et al. [11] recently presented a prospective study of lung transplantation only using initially unacceptable donor lungs, which were improved by ex vivo lung perfusion. In 9 out of 13 organs the median donor $\mathrm{PaO}_{2}$ improved from $216 \mathrm{~mm} \mathrm{Hg}$ (range 133-271) to $466 \mathrm{~mm} \mathrm{Hg}$ (range 434-525). These lungs were successfully transplanted with a 30-day mortality rate of $0 \%$.

Ex vivo lung perfusion is becoming a popular promising method for the extension of preservation time and recuperation of 'low-quality' lungs. Successful transplantation of questionable lungs after ex vivo lung perfusion has recently been reported by several groups worldwide [12]. Cypel et al. [12] used normothermic ex vivo lung perfusion in marginal lungs from 23 donors and in 20 of these lungs, physiological function remained stable during ex vivo perfusion. These lungs were successfully transplanted [13]. Steen et al. [14] proved in animal experiments that the edema developing after $12 \mathrm{~h}$ of cold preservation of the lungs can be prevented by using continuous perfusion for $12 \mathrm{~h}$ or more before the transplant.

We have recently reported on the clinical use of the integrated and portable OCS Lung (Transmedics) for donor lung preservation and transportation. Our goal was to follow a real- 
life scenario that would allow us to realistically estimate the safety and quality of preservation of donor lungs with warm perfusion on the OCS Lung. In this pilot study which included 12 patients, we observed no early mortality or graft loss, times on ventilators and in the intensive-care unit were not excessive, and all patients were discharged from hospital with good lung function after transplantation. Midterm outcomes are encouraging, and spirometry data suggest that patients are in good functional status with healthy and well-functioning lungs [15].

\section{Heart}

Continuous machine perfusion of donor hearts has been proposed as an alternative and superior method of donor heart preservation compared to cold static storage [16]. Continuous ex vivo heart perfusion demonstrated a beneficial influence on aerobic metabolism during the preservation of the donor heart by maintaining cell integrity and vital cell function [17]. Another advantage of continuous coronary organ perfusion is related to the ongoing washout of anaerobic metabolic products, the result of extended ischemia of the organ [18].

Cellular integrity and myocardial recovery may be continuously assessed during ex vivo perfusion by using direct measurement and biochemical analyses ( $\mathrm{pH}$, lactate level, etc.). Moreover, it can be assumed that myocardial viability can be easily assessed using ex vivo cardiac imaging (e.g. ex vivo cardiac MRI). Recently a group from Heidelberg has described a successful ex vivo coronary angiography of a marginal donor heart in the OCS [19]. After having reached the implantation site and having suspected coronary sclerosis, they performed angiography in a donor heart on a catheterization table.

The progress in organ transplantation technologies and the development of ex vivo systems, which mimic the body's physiological state and allow for prolonged preservation, are very promising and can be actively incorporated into ex vivo reconditioning of marginal donor hearts.

\section{Liver}

The first successful human liver transplantation was performed by Starzl et al. [20] using donor liver pretreatment by machine perfusion with diluted, hyperbaric oxygenated blood. However, due to the simplicity of the method, for many years hypothermic, static preservation has been used as the standard for liver preservation [21]. Nevertheless, different approaches regarding optimal temperature, optimal flow rates and perfusion pressures, single or dual vessel (hepatic artery and/or portal vein) perfusion, perfusate oxygenation, and different perfusate compositions are still under investigation [9, 22, 23]. Once these questions have been resolved, ex vivo perfusion systems may play an important role in pretransplant donor liver reconditioning.

\section{Kidneys}

Hypothermic machine perfusion preservation is increasingly used in kidney transplantation as an alternative method to static cold storage for the preservation of grafts obtained from nonoptimal donors. Machine perfusion allows for a wider use of kidneys injured by donor metabolic and hemodynamic instability and results in superior postoperative kidney transplant function compared to cold-stored grafts [24]. Also, several reports indicate that machine perfusion permits an extension of preservation time [25-27]. Moreover, medical reconditioning of machine-perfused kidneys may be used during perfusion through medications administered into the perfusate and modification of flow parameters [28]. 
Fig. 2. Prof. Dr. Rudolf Pichlmayr, during 1968-1997 chief of the Department of Abdominal and Transplantation Surgery, Hannover Medical School, the famous German abdominal surgeon who implemented in 1988 the first ex vivo resection of a hepatic tumor followed by autotransplantation of the organ.

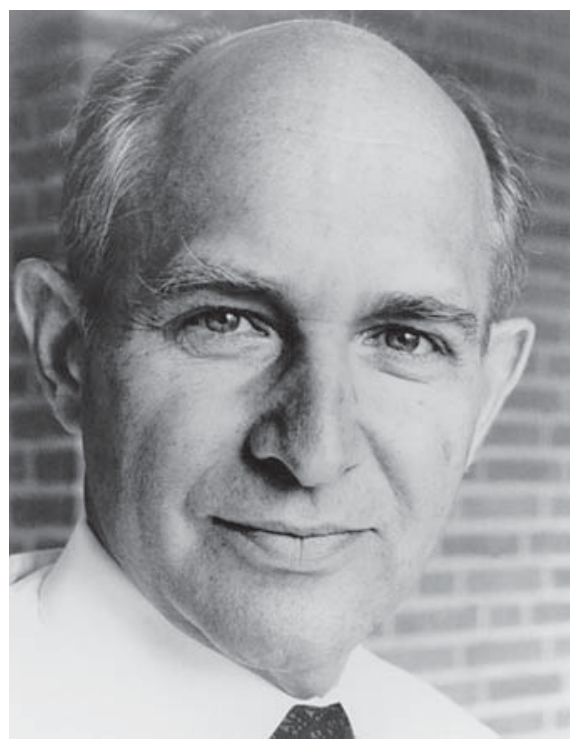

\section{Ex vivo Surgery}

The techniques of ex situ repair of diseased organs with subsequent autotransplantation were developed in experienced centers with large transplantation programs. The advantages of ex vivo surgical resections include optimal visualization and access to all sites, less blood loss and a prolonged period of time for accurate tissue dissection and reconstruction. Pichlmayr et al. [29] (fig. 2) in 1990 identified the following aims for the ex vivo approach: (1) to increase the resectability rate in patients with advanced tumors; (2) to improve the radicality of tumor resections; (3) to avoid the need for organ grafting, and (4) in theory, to allow oncological methods to be used extracorporeally. Ex vivo repair of the abdominal and thoracic organs has been employed since the early 1960s and has been sporadically developed further over the years.

\section{Kidneys}

Surgical management of renovascular hypertension using autotransplantation shows many technical advantages compared to in situ renal revascularization including the option to perform meticulous ex vivo reconstructive surgery. Gallagher et al. [30] described the use of a laparoscopic approach for nephrectomy with the subsequent ex vivo repair of complex renal artery aneurysms involving distal branch arteries. This combination of minimally invasive surgery and ex vivo aneurysm repair resulted in short hospital stays, and good midand long-term results regarding vascular patency and renal function. To bridge the ischemic period in renal autotransplantation during the interruption of the renal circulation, intermediate perfusion with a cold preservation solution is usually performed. Morin et al. [31] used ex vivo kidney perfusion and repair with subsequent autotransplantation and demonstrated the safety of this method even in patients with solitary kidneys.

Liver

In February 1988, Pichlmayr et al. [32] from the Hannover Medical School, Germany, for the first time performed an ex vivo liver resection and autotransplantation in a patient with a huge liver metastasis, which otherwise would have been assessed as truly unresectable (fig. 2). The surgical procedure and the postoperative course were uneventful in this patient. 
Two years later, the same group from Hannover reported on 8 other patients using the same approach [29]. In 3 patients they used in situ hypothermic perfusion in order to operate on a bloodless liver without any time pressure.

Miura et al. [33] described a technique of using liver extraction and autologous reimplantation in order to remove a huge retroperitoneal liposarcoma situated behind the liver. Recently, a group from the Xinjiang Medical University in China reported for the first time an ex vivo liver resection for alveolar echinococcosis [34]. In their center they performed seven liver transplantations in patients with end-stage alveolar echinococcosis; after having refined the surgical techniques, they were able to address the same pathology using an ex vivo resection with partial liver autotransplantation.

\section{Intestinal and Multivisceral Autotransplantation}

Tumors that involve the mesenteric vascular pedicle are often considered unresectable. The development of intestinal transplantation opened new curative alternatives for these patients. However, allotransplantation of the intestine is not a common procedure due to high rejection rates. On the other hand, advances in intestinal transplantation expand previous boundaries to include ex vivo resection and autotransplantation.

Recently a group from the University of Miami reported their largest series of ex vivo resections on 10 patients with neoplasms of the base of the mesentery that were considered nonresectable using conventional resection [35]. The patients were originally referred to the Department of Intestinal or Multivisceral Allotransplantation. After extensive evaluation, all patients underwent abdominal visceral exenteration, ex vivo resection and intestinal autotransplantation. The authors concluded that intestinal/multivisceral autotransplantation is a potentially valuable option for some unresectable neoplasms of the root of the mesentery [35].

The method of multivisceral and intestinal autotransplantation appears to be feasible and effective in experienced hands in a well-defined clinical situation.

\section{Heart}

The method of autotransplantation of the heart was first used for treatment of Prinzmetal's angina. However, due to poor clinical outcomes, this procedure was soon abandoned $[36,37]$. The first human cardiac explantation, ex vivo surgery and autotransplantation was described by Cooley et al. [38] in 1985. They introduced this innovative operation method in order to resect a rare paraganglioma located intrapericardially. Although the results were unfavorable in this case, Cooley et al. concluded that the technique of autotransplantation deserves consideration for resecting large tumors that encroach upon the heart. His student, Dr. Reardon, was the first surgeon who successfully used autotransplantation and ex vivo tumor resection for primary malignant heart tumors [39]. Reardon and his colleague [40] successfully developed the methods of cardiac explantation, preservation and autotransplantation further.

This technique was successfully used for surgical resection of malignant or complex primary left-heart tumors as well as benign tumors such atrial myxomas. In 2008 Reardon and his colleagues [41] reported on 20 patients who underwent ex vivo heart tumor resections. In this series the mean survival of all patients with sarcoma was 22 months. The authors concluded that complete ex vivo resection improves survival rates among patients who have cardiac sarcomas. However, our group demonstrated that in some specific cases only partial ex situ techniques can be applied to resect tumors involving the posterior side of the heart $[42,43]$.

\section{Lungs}

The first successful clinical ex situ lung resection followed by autotransplantation was described by Zhang et al. [44] in 1998. In 2000, a group from the University of Regensburg 
applied ex situ tumor resection and autotransplantation on a patient with left-sided nonsmall-cell lung cancer invading the main bronchus, pulmonary artery and left atrium. After extended pneumonectomy, the lower lobe was divided ex situ from the upper lobe and reimplanted with an anastomosis of the lower pulmonary vein to the left auricle [45].

Our group recently described an experimental setting using the OCS system for ex vivo pulmonary surgery. During ex vivo resection and reconstructive surgery, perfusion and ventilation of the lung were maintained by OCS under physiological conditions. This ex vivo approach using the OCS preservation system facilitates more accurate conservation of the lung during surgery, ex situ testing of reconstruction in terms of blood or air leakage, and, furthermore, the combination of ex vivo surgery with topical ex situ medical treatment of the diseased tissue [46].

\section{Ex vivo Medical Treatment}

Once organs are retrieved from the body, ex vivo treatment especially during machine perfusion becomes possible [6]. Perfusion under physiological conditions providing oxygen and other metabolic substrates facilitates organ function recovery. Additionally, ex vivo perfusion may be used for topical administration of drugs in order to reduce injury and to restore organ function. Different drugs, alone or in combination, can be used during ex vivo organ perfusion to address a specific type of injury. Examples would be: (1) explantation of an injured nonoxygenating lung to reduce the shunt volume and to improve oxygenation of the body, (2) ex vivo lung treatment and (3) subsequent autotransplantation of the organ once it has regained a good medical condition. In this case the body is protected from the detrimental effects of a diseased organ, while simultaneously the organ treatment is effectively addressed locoregionally in an ex vivo setting. Thus, adverse effects on the whole body are avoided. Locoregional treatment may include direct organ administration of antibacterial, antifungal and antiviral agents, and vasodilating and anti-inflammatory medication. Also, adjuvant therapy including chemo- or radiotherapy could be used ex situ in order to locally address the tumor in combination with (or without) ex vivo tumor resection. The great advantage in this case is that the ex situ treatment can be applied locally in high dosages without harming other organs and tissues (fig. 3).

\section{Locoregional Chemotherapy}

Although Pichlmayr et al. [29] in 1990 in their article regarding extracorporeal liver resection suggested using oncological treatment methods ex vivo, so far locoregional chemotherapy has only been performed using isolated organ perfusion in situ. In 2002, our group described a hyperthermic $\left(41^{\circ} \mathrm{C}\right)$ isolated lung perfusion technique with high doses of cisplatin for the treatment of surgically relapsing or unresectable lung sarcoma metastases [47]. The isolated lung perfusion was carried out in situ following metastasectomy for 20$40 \mathrm{~min}$ at a rate of $0.3-0.5 \mathrm{liter} / \mathrm{min}$ and at an inflow temperature of $41^{\circ} \mathrm{C}$ or higher. At a 12 month follow-up, 3 patients were alive and disease-free and 1 had died from a cerebral metastasis without evidence of local recurrence [47].

After extensive experimental research, isolated in situ organ perfusion using chemotherapeutic drugs has now become a feasible clinical technique [48-50]. In different clinical studies, isolated organ perfusion procedures are generally well tolerated and reproducible offering a valid clinical model for further investigation of combined chemotherapy and surgery in patients with pulmonary metastases $[51,52]$.

With the development of modern ex vivo perfusion systems, it can be assumed that adjuvant therapy may be performed more safely once the affected organ is completely explanted 


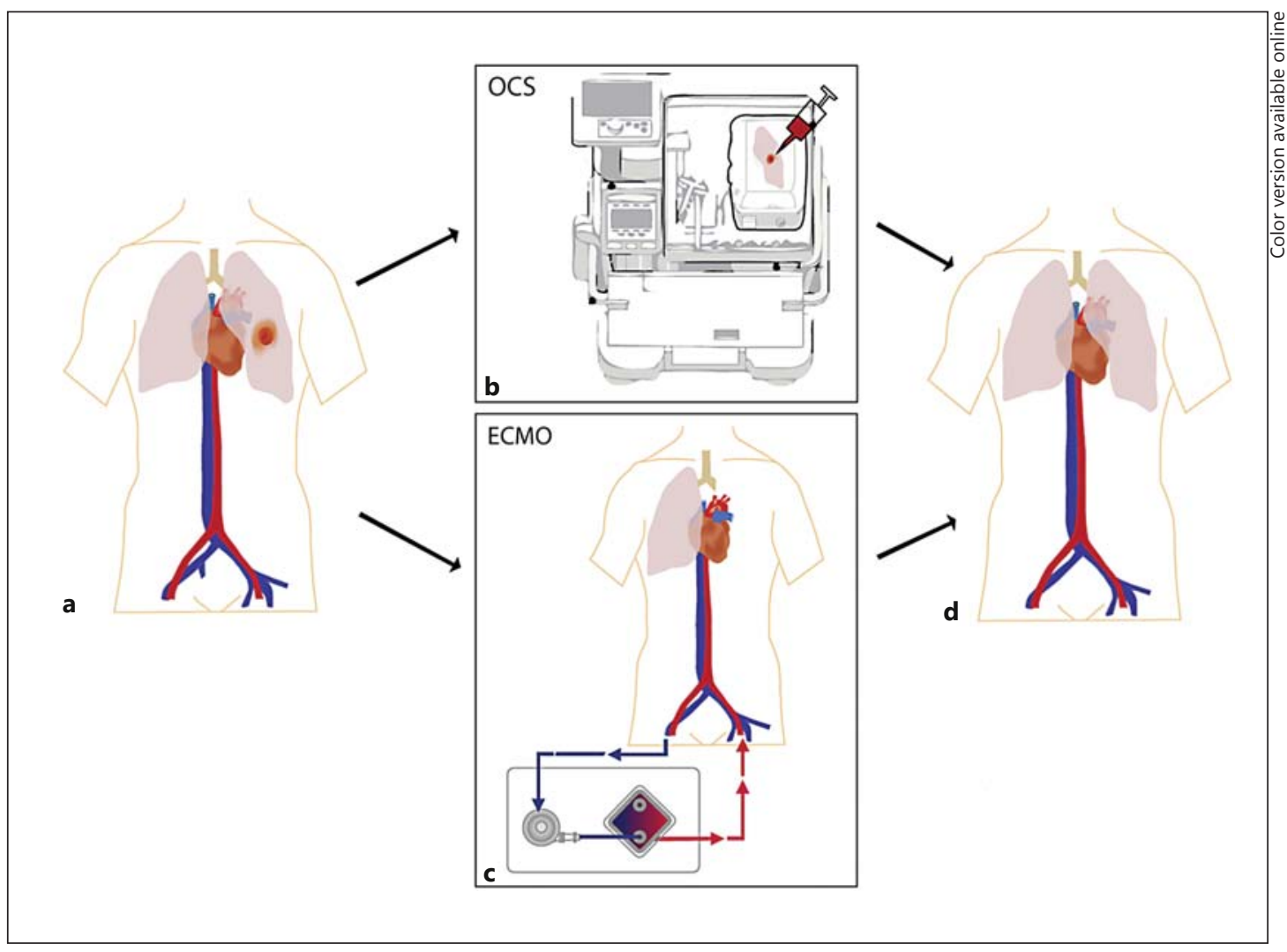

Fig. 3. Artistic illustration depicting the concept of ex vivo organ treatment using the lungs as a template. a Diseased left lung. $\mathbf{b}$ Ex vivo treatment and recovery of the lung in the OCS. c Connection of the body to the extracorporeal membrane oxygenation (ECMO) support system. d Autotransplantation of the cured lung.

from the body. Moreover, the availability of a closed perfusion system with the possibility of exchanging the perfusate solution or influencing different parameters such as the temperature of the flow rate makes it even easier and safer to use an ex vivo perfusion system [53].

\section{Ex vivo Gene and Cell Therapies}

Recently, modern approaches such as gene or cell therapies have been proposed for ex vivo organ recovery. Cypel et al. [54] applied gene therapy in 10 human lungs from multiorgan donors that were determined to be clinically unsuitable for transplantation. These organs were maintained for $12 \mathrm{~h}$ at body temperature in an ex vivo lung perfusion system using an intra-airway delivery of the AdhIL-10 gene therapy. AdhIL-10-treated lungs showed significant improvement in function (arterial oxygen pressure and pulmonary vascular resistance) when compared to controls, a favorable shift from pro-inflammatory to anti-inflammatory cytokine expression and recovery of alveolar-blood barrier integrity [54].

Lee et al. [55] completed an experimental study on the therapeutic capacity of human mesenchymal stem cells to restore alveolar epithelial fluid transport and lung fluid balance after acute lung injury in an ex vivo perfused human lung preparation. The authors demonstrated that treatment with allogeneic human mesenchymal stem cells reduced pulmonary edema, improved lung endothelial barrier integrity and normalized alveolar epithelial fluid transport in an ex vivo perfused human lung. These experiments underline the feasibility of modern treatment options including gene and cell therapy to treat different pathologies addressing the affected organ ex vivo. 


\section{Future Considerations}

(1) Modern life-support systems, which have been developed substantially in recent years, improved the survival rate of patients with failing organs and allowed a temporary substitution of an organ function. These technologies can bridge the time until the organs of the patients completely or partially recover.

(2) The development of modern organ perfusion systems, in which biomechanical and biophysiological conditions can be adjusted to mimic the physiological environment, allows safe ex vivo organ preservation for longer periods of time and reduces ischemic time.

(3) Ex vivo organ preservation permits the exact evaluation of organ function and allows the application of ex situ diagnoses including exact topographical assessment of an isolated organ using ex vivo imaging (e.g. ex vivo MRI, CT, angiography or 3D echography).

(4) Ex vivo organ perfusion providing oxygen and nutrients under physiological conditions and removing toxic metabolic products permits improvement of organ function and organ reconditioning. This method allows the recovery of organs, which until recently, have been rejected for transplant.

(5) Ex vivo organ perfusion permits direct administration of drugs to the organ by injecting them into tubes connected to the organ (e.g. endovascular or endobronchial), thus omitting systemic administration of drugs and their adverse effects on other organs and systems. Moreover, explanted organs can be exposed to radiotherapy in order to avoid systemic exposure to radiation.

(6) Even though there have been several sporadic attempts at ex vivo surgical resection and correction of different organs in treating nonoperable patients, the optimal preservation of affected organs in OCSs opens new possibilities for ex vivo surgery. Although ex vivo surgical resection has mainly been used in oncological surgery, improvement in ex vivo organ preservation and recovery may expand the indications for ex vivo surgery to other complicated pathologies (e.g. complex congenital malformations).

(7) The combination of (a) ex vivo organ perfusion and storage, (b) ex vivo exact topographical diagnosis, (c) ex vivo locoregional medical treatment including the possibility of ex situ radiotherapy and (d) the possibility of ex vivo surgical correction can expand the medical possibilities in the treatment of failing organs, thus improving morbidity and mortality. The ex vivo approach offers prospects for applying the above-mentioned methods to most human organs, when traditional methods cannot offer treatment.

Ex vivo therapies open new horizons in the treatment of end-stage organ pathologies. Further experimental and clinical research is needed to develop ex vivo approaches regarding organ preservation, topographical diagnosis, surgical techniques, pharmacokinetics as well as new methods of treatment including gene and cell therapies and tissue engineering.

\section{References}

$\checkmark 1$ Pomfret EA, Sung RS, Allan J, Kinkhabwala M, Melancon JK, Roberts JP: Solving the organ shortage crisis: the 7 th annual American Society of Transplant Surgeons' State-of-the-Art Winter Symposium. Am J Transplant 2008;8:745-752.

2 Watson CJ, Dark JH: Organ transplantation: historical perspective and current practice. Br J Anaesth 2012; 108(suppl 1):i29-i42.

-3 Diaz-Guzman E, Hoopes CW, Zwischenberger JB: The evolution of extracorporeal life support as a bridge to lung transplantation. ASAIO J 2013;59:3-10.

4 Fuehner T, Kuehn C, Hadem J, Wiesner O, Gottlieb J, Tudorache I, et al: Extracorporeal membrane oxygenation in awake patients as bridge to lung transplantation. Am J Respir Crit Care Med 2012;185:763-768.

-5 Olsson KM, Simon A, Strueber M, Hadem J, Wiesner O, Gottlieb J, et al: Extracorporeal membrane oxygenation in nonintubated patients as bridge to lung transplantation. Am J Transplant 2010;10:2173-2178. 
6 Van Raemdonck D, Neyrinck A, Rega F, Devos T, Pirenne J: Machine perfusion in organ transplantation: a tool for ex-vivo graft conditioning with mesenchymal stem cells? Curr Opin Organ Transplant 2013;18:24-33.

7 Sanchez PG, Bittle GJ, Burdorf L, Pierson RN 3rd, Griffith BP: State of art: clinical ex vivo lung perfusion: rationale, current status, and future directions. J Heart Lung Transplant 2012;31:339-348.

-8 Souilamas R, Souilamas JI Jr, Saueressig M, Briot R: Advanced normothermic ex vivo lung maintenance using the mobile Organ Care System. J Heart Lung Transplant 2011;30:847-848.

-9 Monbaliu D, Brassil J: Machine perfusion of the liver: past, present and future. Curr Opin Organ Transplant 2010;15:160-166.

10 Ingemansson R, Eyjolfsson A, Mared L, Pierre L, Algotsson L, Ekmehag B, et al: Clinical transplantation of initially rejected donor lungs after reconditioning ex vivo. Ann Thorac Surg 2009;87:255-260.

$>11$ Aigner C, Slama A, Hotzenecker K, Scheed A, Urbanek B, Schmid W, et al: Clinical ex vivo lung perfusion pushing the limits. Am J Transplant 2012;12:1839-1847.

12 Van Raemdonck D, Neyrinck A, Cypel M, Keshavjee S: Ex-vivo lung perfusion. Transpl Int 2014, Epub ahead of print.

-13 Van Raemdonck D: Thoracic organs: current preservation technology and future prospects; part 1: lung. Curr Opin Organ Transplant 2010;15:150-155.

14 Steen S, Liao Q, Wierup PN, Bolys R, Pierre L, Sjoberg T: Transplantation of lungs from non-heart-beating donors after functional assessment ex vivo. Ann Thorac Surg 2003;76:244-252; discussion 52.

15 Warnecke G, Moradiellos J, Tudorache I, Kuhn C, Avsar M, Wiegmann B, et al: Normothermic perfusion of donor lungs for preservation and assessment with the Organ Care System Lung before bilateral transplantation: a pilot study of 12 patients. Lancet 2012;380:1851-1858.

-16 Collins MJ, Moainie SL, Griffith BP, Poston RS: Preserving and evaluating hearts with ex vivo machine perfusion: an avenue to improve early graft performance and expand the donor pool. Eur J Cardiothorac Surg 2008;34: 318-325.

17 Ozeki T, Kwon MH, Gu J, Collins MJ, Brassil JM, Miller MB Jr, et al: Heart preservation using continuous ex vivo perfusion improves viability and functional recovery. Circ J 2007;71:153-159.

-18 Rosenbaum DH, Peltz M, DiMaio JM, Meyer DM, Wait MA, Merritt ME, et al: Perfusion preservation versus static preservation for cardiac transplantation: effects on myocardial function and metabolism. J Heart Lung Transplant 2008;27:93-99.

19 Ghodsizad A, Bordel V, Ungerer M, Karck M, Bekeredjian R, Ruhparwar A: Ex vivo coronary angiography of a donor heart in the organ care system. Heart Surg Forum 2012;15:E161-E163.

-20 Starzl TE, Groth CG, Brettschneider L, Moon JB, Fulginiti VA, Cotton EK, et al: Extended survival in 3 cases of orthotopic homotransplantation of the human liver. Surgery 1968;63:549-563.

21 Franco-Gou R, Mosbah IB, Serafin A, Abdennebi HB, Rosello-Catafau J, Peralta C: New preservation strategies for preventing liver grafts against cold ischemia reperfusion injury. J Gastroenterol Hepatol 2007;22:11201126.

22 Hessheimer AJ, Fondevila C, Garcia-Valdecasas JC: Extracorporeal machine liver perfusion: are we warming up? Curr Opin Organ Transplant 2012;17:143-147.

23 Vogel T, Brockmann JG, Friend PJ: Ex-vivo normothermic liver perfusion: an update. Curr Opin Organ Transplant 2010;15:167-172.

-24 Moers C, Smits JM, Maathuis MH, Treckmann J, van Gelder F, Napieralski BP, et al: Machine perfusion or cold storage in deceased-donor kidney transplantation. N Engl J Med 2009;360:7-19.

25 Nicholson ML, Hosgood SA, Metcalfe MS, Waller JR, Brook NR: A comparison of renal preservation by cold storage and machine perfusion using a porcine autotransplant model. Transplantation 2004;78:333-337.

-26 Brook NR, Waller JR, Nicholson ML: Nonheart-beating kidney donation: current practice and future developments. Kidney Int 2003;63:1516-1529.

27 Schilling M, Saunder A, Southard JH, Belzer FO: Long-term renal preservation and prevention of acute tubular necrosis by inhibition of arachidonate metabolism. Swiss Surg 1995;4:205-209.

28 Polyak MM, Arrington BO, Kapur S, Stubenbord WT, Kinkhabwala M: Donor treatment with phentolamine mesylate improves machine preservation dynamics and early renal allograft function. Transplantation 2000; 69:184-186.

29 Pichlmayr R, Grosse H, Hauss J, Gubernatis G, Lamesch P, Bretschneider HJ: Technique and preliminary results of extracorporeal liver surgery (bench procedure) and of surgery on the in situ perfused liver. Br J Surg 1990; $77: 21-26$.

-30 Gallagher KA, Phelan MW, Stern T, Bartlett ST: Repair of complex renal artery aneurysms by laparoscopic nephrectomy with ex vivo repair and autotransplantation. J Vasc Surg 2008;48:1408-1413.

-31 Morin J, Chavent B, Duprey A, Albertini JN, Favre JP, Barral X: Early and late results of ex vivo repair and autotransplantation in solitary kidneys. Eur J Vasc Endovasc Surg 2012;43:716-720.

-32 Pichlmayr R, Bretschneider HJ, Kirchner E, Ringe B, Lamesch P, Gubernatis G, et al: Ex situ operation on the liver. A new possibility in liver surgery. Langenbecks Arch Chir 1988;373:122-126.

-33 Miura K, Sato Y, Kokai H, Hara Y, Kobayashi T, Oya H, et al: Liver transplantation surgical techniques for extensive retroperitoneal tumor with major blood vessel involvement: a case report. Transplant Proc 2012; 44:579-580.

-34 Wen H, Dong JH, Zhang JH, Zhao JM, Shao YM, Duan WD, et al: Ex vivo liver resection followed by autotransplantation for end-stage hepatic alveolar echinococcosis. Chin Med J 2011;124:2813-2817. 
-35 Tzakis AG, Pararas NB, Tekin A, Gonzalez-Pinto I, Levi D, Nishida S, et al: Intestinal and multivisceral autotransplantation for tumors of the root of the mesentery: long-term follow-up. Surgery 2012;152:82-89.

-36 Clark DA, Quint RA, Mitchell RL, Angell WW: Coronary artery spasm. Medical management, surgical denervation, and autotransplantation. J Thorac Cardiovasc Surg 1977;73:332-339.

-37 Bertrand ME, Lablanche JM, Tilmant PY, Ducloux G, Warembourg H Jr, Soots G: Complete denervation of the heart (autotransplantation) for treatment of severe, refractory coronary spasm. Am J Cardiol 1981;47:13751378.

38 Cooley DA, Reardon MJ, Frazier OH, Angelini P: Human cardiac explantation and autotransplantation: application in a patient with a large cardiac pheochromocytoma. Tex Heart Inst J 1985;12:171-176.

-39 Reardon MJ, DeFelice CA, Sheinbaum R, Baldwin JC: Cardiac autotransplant for surgical treatment of a malignant neoplasm. Ann Thorac Surg 1999;67:1793-1795.

40 Conklin LD, Reardon MJ: Autotransplantation of the heart for primary cardiac malignancy: development and surgical technique. Tex Heart Inst J 2002;29:105-108; discussion 108.

-41 Blackmon SH, Patel AR, Bruckner BA, Beyer EA, Rice DC, Vaporciyan AA, et al: Cardiac autotransplantation for malignant or complex primary left-heart tumors. Tex Heart Inst J 2008;35:296-300.

42 Kallenbach K, Cremer J, Haverich A: Partial ex situ surgery of the heart. J Thorac Cardiovasc Surg 2002;123: 577-578.

43 Kallenbach K, Haverich A: Explantation of the heart for resection of primary cardiac tumors can be avoided by partial ex situ heart surgery. Thorac Cardiovasc Surg 2003;51:293-294.

44 Zhang G, Li M, Yan G, Liu J, Jiang G: Lung autotransplantation technique in the treatment of stage III bronchogenic carcinoma (in Chinese). Zhonghua Wai Ke Za Zhi 1998;36:158-160.

-45 Hendrix H, Schmitt J, Aebert H: Ex situ sleeve resection and autotransplantation of the left lower pulmonary lobe after extended pneumonectomy (in German). Chirurg 2000;71:820-823.

46 Kruger M, Zinne N, Hoffler H, Zhang R, Kropivnitskaja I, Schmitto J, et al: Ex situ tracheobronchoplastic operations using the organ care system (in German). Chirurg 2013;84:208-213.

-47 Schroder C, Fisher S, Pieck AC, Muller A, Jaehde U, Kirchner H, et al: Technique and results of hyperthermic (41 degrees $C$ ) isolated lung perfusion with high-doses of cisplatin for the treatment of surgically relapsing or unresectable lung sarcoma metastasis. Eur J Cardiothorac Surg 2002;22:41-46.

48 Krementz ET: Lucy Wortham James lecture. Regional perfusion. Current sophistication, what next? Cancer 1986;57:416-432.

49 Johnston MR, Christensen CW, Minchin RF, Rickaby DA, Linehan JH, Schuller HM, et al: Isolated total lung perfusion as a means to deliver organ-specific chemotherapy: long-term studies in animals. Surgery 1985;98: 35-44.

50 Putnam JB Jr: New and evolving treatment methods for pulmonary metastases. Semin Thorac Cardiovasc Surg 2002;14:49-56.

51 Grootenboers MJ, Schramel FM, van Boven WJ, van Putte BP, Hendriks JM, Van Schil PE: Re-evaluation of toxicity and long-term follow-up of isolated lung perfusion with melphalan in patients with resectable pulmonary metastases: a phase I and extension trial. Ann Thorac Surg 2007;83:1235-1236.

52 Van Schil PE, Hendriks JM, van Putte BP, Stockman BA, Lauwers PR, Ten Broecke PW, et al: Isolated lung perfusion and related techniques for the treatment of pulmonary metastases. Eur J Cardiothorac Surg 2008; 33:487-496.

53 Cypel M, Keshavjee S: The clinical potential of ex vivo lung perfusion. Expert Rev Respir Med 2012;6:27-35.

54 Cypel M, Liu M, Rubacha M, Yeung JC, Hirayama S, Anraku M, et al: Functional repair of human donor lungs by IL-10 gene therapy. Sci Transl Med 2009;1:4ra9.

55 Lee JW, Fang X, Gupta N, Serikov V, Matthay MA: Allogeneic human mesenchymal stem cells for treatment of E. coli endotoxin-induced acute lung injury in the ex vivo perfused human lung. Proc Natl Acad Sci USA 2009; 106:16357-16362. 